Thielen, Marc

\title{
Männlichkeit verpflichtet. Die pädagogische Bearbeitung randständiger Männlichkeit im Zuge der Herstellung von Ausbildungsreife in der \\ Berufsvorbereitung
}

Budde, Jürgen [Hrsg.]; Thon, Christine [Hrsg.]; Walgenbach, Katharina [Hrsg.]: Männlichkeiten Geschlechterkonstruktionen in pädagogischen Institutionen. Opladen ; Berlin ; Toronto : Verlag Barbara Budrich 2014, S. 171-184. - (Jahrbuch der Frauen- und Geschlechterforschung in der Erziehungswissenschaft; 10)

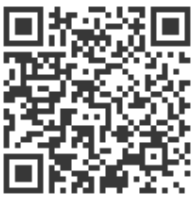

\section{Quellenangabe/ Reference:}

Thielen, Marc: Männlichkeit verpflichtet. Die pädagogische Bearbeitung randständiger Männlichkeit im Zuge der Herstellung von Ausbildungsreife in der Berufsvorbereitung - In: Budde, Jürgen [Hrsg.]; Thon, Christine [Hrsg.]; Walgenbach, Katharina [Hrsg.]: Männlichkeiten - Geschlechterkonstruktionen in pädagogischen Institutionen. Opladen ; Berlin ; Toronto : Verlag Barbara Budrich 2014, S. 171-184 URN: urn:nbn:de:0111-pedocs-122160 - DOI: 10.25656/01:12216

https://nbn-resolving.org/urn:nbn:de:0111-pedocs-122160

https://doi.org/10.25656/01:12216

in Kooperation mit / in cooperation with:

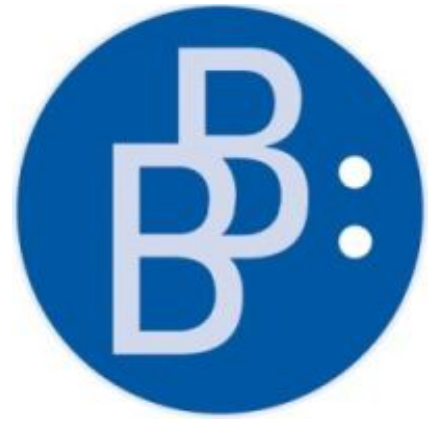

https://www.budrich.de

\section{Nutzungsbedingungen}

Gewährt wird ein nicht exklusives, nicht übertragbares, persönliches und beschränktes Recht auf Nutzung dieses Dokuments. Dieses Dokument ist ausschließlich für den persönlichen, nicht-kommerziellen Gebrauch bestimmt. Die Nutzung stellt keine Übertragung des Eigentumsrechts an diesem Dokument dar und gilt vorbehaltlich der folgenden Einschränkungen Auf sämtlichen Kopien dieses Dokuments müssen alle Urheberrechtshinweise und sonstigen Hinweise auf gesetzlichen Schutz beibehalten werden. Sie dürfen dieses Dokument nicht in irgendeiner Weise abändern, noch dürfen Sie dieses Dokument für öffentliche oder kommerzielle Zwecke vervielfältigen, öffentlich ausstellen, aufführen, vertreiben oder anderweitig nutzen.

Mit der Verwendung dieses Dokuments erkennen Sie die Nutzungsbedingungen an.

\section{Terms of use}

We grant a non-exclusive, non-transferable, individual and limited right to using this document.

This document is solely intended for your personal, non-commercial use. Use of this document does not include any transfer of property rights and it is conditional to the following limitations: All of the copies of this documents must retain all copyright information and other information regarding legal protection. You are not allowed to alter this document in any way, to copy it for public or commercial purposes, to exhibit the document in public, to perform, distribute or otherwise use the document in public.

By using this particular document, you accept the above-stated conditions of use.

\section{Kontakt / Contact:}

peDOCS

DIPF | Leibniz-Institut für Bildungsforschung und Bildungsinformation Informationszentrum (IZ) Bildung

E-Mail: pedocs@dipf.de

Internet: www.pedocs.de

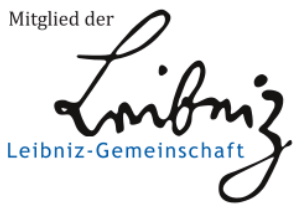


Männlichkeiten 
Jahrbuch

Frauen- und Geschlechterforschung

in der Erziehungswissenschaft

Redaktion

Jürgen Budde

Vera Moser

Barbara Rendtorff

Christine Thon

Katharina Walgenbach

Beirat

Birgit Althans

Sabine Andresen

Eva Breitenbach

Rita Casale

Bettina Dausien

Isabell Diehm

Hannelore Faulstich-Wieland

Edgar Forster

Edith Glaser

Carola Iller

Marita Kampshoff

Margret Kraul

Andrea Liesner

Susanne Maurer

Astrid Messerschmidt

Inga Pinhard

Annedore Prengel

Folge 10/2014 
Jürgen Budde

Christine Thon

Katharina Walgenbach (Hrsg.)

\section{Männlichkeiten}

Geschlechterkonstruktionen in pädagogischen Institutionen

Verlag Barbara Budrich

Opladen, Berlin \& Toronto 2014 
Bibliografische Information der Deutschen Nationalbibliothek

Die Deutsche Nationalbibliothek verzeichnet diese Publikation in der Deutschen

Nationalbibliografie; detaillierte bibliografische Daten sind im Internet über

http://dnb.d-nb.de abrufbar.

Gedruckt auf säurefreiem und alterungsbeständigem Papier.

Alle Rechte vorbehalten.

(C) 2014 Verlag Barbara Budrich, Opladen, Berlin \& Toronto

www.budrich-verlag.de

\section{ISBN 978-3-8474-0168-1 (Paperback)}

eISBN 978-3-8474-0440-8 (eBook)

Das Werk einschließlich aller seiner Teile ist urheberrechtlich geschützt. Jede Verwertung außerhalb der engen Grenzen des Urheberrechtsgesetzes ist ohne Zustimmung des Verlages unzulässig und strafbar. Das gilt insbesondere für Vervielfältigungen, Übersetzungen, Mikroverfilmungen und die Einspeicherung und Verarbeitung in elektronischen Systemen.

Typographisches Lektorat: Ulrike Weingärtner, Gründau

Umschlaggestaltung: disegno visuelle kommunikation, Wuppertal - www.disenjo.de Druck: paper \& tinta, Warschau

Printed in Europe 
Inhalt

\section{Einleitung}

Jürgen Budde, Christine Thon \& Katharina Walgenbach

Männlichkeiten - Geschlechterkonstruktionen in pädagogischen

Institutionen

\section{Essay}

Wayne Martino

Teaching boys in neoliberal and post-feminist times: Feminization and the question of re-masculinization in the education system and policy field.

\section{Männlichkeit als professionelle Ressource in Institutionen}

Eva Breitenbach \& Ilse Bürmann

Heilsbringer oder Erlösungssucher? Befunde und Thesen zur

Problematik von Männern in frühpädagogischen Institutionen

Tim Rohrmann

Männer in Kitas: Zwischen Idealisierung und Verdächtigung..... 67

Wiebke Bobeth-Neumann

„Man(n) wird da so hineingelobt“" - Ko-Konstruktion von

Männlichkeit und Aufstieg ins Schulleitungsamt an Grundschulen 85

Männlichkeit als Referenzpunkt für die Entwicklung institutioneller Strukturen

Jürgen Budde, Katja Kansteiner \& Andrea Bossen

Männlichkeitskonzeptionen in geschlechterdifferenzierender

Schulkultur 
Rosemarie Godel-Gaßner \& Rafael Frick

Übergänge auf Jungenschulen: Schulwahlmotive von Eltern

\section{Pädagogische Orientierungen an Männlichkeiten in Institutionen}

Ruth Michalek, Thomas Fuhr \& Gudrun Schönknecht

Der Zusammenhang von Männlichkeitskonstruktionen mit der Lernund Leistungsmotivation bei Jungen. Ergebnisse einer empirischen

Studie .....

Marc Thielen

Männlichkeit verpflichtet.Die pädagogische Bearbeitung

randständiger Männlichkeit im Zuge der Herstellung von

Ausbildungsreife in der Berufsvorbereitung

\section{Offener Teil}

Kim-Patrick Sabla \& Julia Rohde

Vergeschlechtlichte Professionalität - Zuschreibungen einer

,gelingenden“ Praxis qua Geschlecht

Jeannette Windheuser

Die symbolische und generationale Ordnung der sexuellen Gewalt in der Missbrauchs-Debatte

\section{Tagungsbericht}

Natascha Compes

Geschlecht in gesellschaftlichen Transformationsprozessen 


\section{Rezension}

Detlef Pech

Sammelrezension: Jungen, Probleme, Männer, Krise, Pädagogik. Eine Aneinanderreihung.

Autor innenverzeichnis 


\title{
Männlichkeit verpflichtet. Die pädagogische Bearbeitung randständiger Männlichkeit im Zuge der Herstellung von Ausbildungsreife in der Berufsvorbereitung
}

\author{
Marc Thielen
}

\begin{abstract}
Ungeachtet des demographischen Wandels und des prognostizierten Fachkräftemangels gelingt Jugendlichen ohne einen mittleren Schulabschluss nach der allgemeinbildenden Schule nur selten ein direkter Übergang in eine vollqualifizierende Berufsausbildung. Ausbildungsbetriebe aber auch Lehrkräfte begründen dies häufig mit einer unzureichenden Ausbildungsreife der Schulabgänger/-innen (vgl. DIHK 2012; Müller/Rebmann 2008). Berufsvorbereitende Maßnahmen für Jugendliche ohne Ausbildungsplatz zielen auf die Förderung von Ausbildungsreife, um den Übergang in Ausbildung zu erleichtern. Die entsprechenden pädagogischen Praktiken fokussieren nicht zuletzt auch auf die Geschlechterkonstruktionen der teilnehmenden Jugendlichen, deren Berufswahlverhalten von geschlechtsbezogenen Rollenerwartungen abhängt (vgl. Beicht/Walden 2012). In Maßnahmen, die auf klassische ,Männerberufe" vorbereiten, steht die Männlichkeit der Teilnehmenden im Fokus. Dies verdeutlicht der vorliegende Beitrag anhand einer ethnographischen Untersuchung zum institutionellen Alltag einer Berufsvorbereitungsklasse an einer berufsbildenden Schule. Die ausnahmslos aus Jungen bestehende Lerngruppe wird auf eine Berufsausbildung in der Lagerlogistik vorbereitet einem Berufsfeld, das angesichts der körperlich schweren Arbeit als männlich dominiert gilt. Im Unterricht lassen sich pädagogische Praktiken beobachten, die explizit an die Maskulinität der Schüler appellieren und auf die Ausbildung männlicher Härte zielen, da diese für die Bewältigung des rauen und körperlich belastenden Arbeitsalltags im Berufsfeld als funktional betrachtet wird. Folglich geht die Förderung von Ausbildungsreife mit der Reproduktion traditioneller Männlichkeitserwartungen einher. Bevor dies anhand eines exemplarischen Blicks auf die Mikroebene des unterrichtlichen Alltags in einer Berufsvorbereitungsklasse verdeutlicht wird, erfolgen zunächst Erläuterungen zum institutionellen Kontext der Berufsvorbereitung.
\end{abstract}


Daran anknüpfend wird die Bedeutung von Männlichkeit für den institutionellen Alltag männlichkeitstheoretisch reflektiert. Sodann werden Anlage und Methode der dem Beitrag zugrunde liegenden ethnographischen Studie diskutiert und ausgewählte Ergebnisse präsentiert.

\section{Schulische Berufsvorbereitung als pädagogisches Arrangement zur Förderung von Ausbildungsreife}

Berufsvorbereitungsklassen an berufsbildenden Schulen sind für Jugendliche ohne Ausbildungsplatz vorgesehen, die noch schulpflichtig sind. Die Teilnahme an den in den Bundesländern unterschiedlich bezeichneten Bildungsgängen ist also keineswegs freiwillig. Meist müssen sich die Jugendlichen bei der Anmeldung für ein bestimmtes Berufsfeld entscheiden und werden der darauf spezialisierten berufsbildenden Schule zugewiesen (vgl. Schroeder/Thielen 2009). Da das Berufswahlverhalten von Jugendlichen noch immer stark von geschlechtsbezogenen Erwartungen abhängt, kommt es je nach Berufsfeld $\mathrm{zu}$ deutlichen Überrepräsentanzen von Schülerinnen (z.B. im Bereich Hauswirtschaft) oder Schülern (z.B. in der Metall- und Holztechnik). Unabhängig vom Geschlecht steht schulisch gering qualifizierten Jugendlichen angesichts der Segmentierung des Ausbildungssystems und gestiegener Anforderungen in vielen Ausbildungsberufen nur eine begrenzte Auswahl an Berufen offen, attraktive Berufe werden von Jugendlichen mit mittleren und höheren Schulabschlüssen dominiert (vgl. Baethge 2010). Neben der Vorbereitung auf eine Berufsausbildung bietet der berufsvorbereitende Bildungsgang die Möglichkeit, einen Schulabschluss nachzuholen. Die Schülerschaft hat überwiegend eine geringe schulische Qualifikation (Hauptschulabschluss oder kein Schulabschluss) und einen ungünstigen sozialen Hintergrund: Die Eltern verfügen meist über keinen Berufsabschluss und gehen gering qualifizierten Tätigkeiten nach. Zudem ist eine Überrepräsentanz von Jugendlichen mit Migrationshintergrund zu verzeichnen (vgl. Beicht/Eberhard 2013: 15).

Aus Sicht von Ausbildungsbetrieben und Arbeitgebervertretern begründet sich die Teilnahme an der Berufsvorbereitung in einem besonderen berufspädagogischen Förderbedarf: Schulisch gering qualifizierten Jugendlichen wird häufig eine unzureichende Ausbildungsreife attestiert (vgl. Frieling/Ulrich 2013: 78ff.). Jenes berufsbildungswissenschaftlich umstrittene Konstrukt benennt Mindestvoraussetzungen, die Jugendliche für das erfolgreiche Absolvieren einer Berufsausbildung benötigen. Die Bundesagentur für Arbeit (2009) zählt hierzu schulische Basiskenntnisse, psychologische Leistungsmerkmale, physische Merkmale, Berufswahlreife sowie psychologische Merkmale des Arbeitsverhaltens und der Persönlichkeit. Die zuletzt genannte 
Dimension von Ausbildungsreife ist mit Blick auf den vorliegenden Beitrag besonders interessant: Subsumiert wird hierunter beispielsweise Durchhaltevermögen, Konfliktfähigkeit, Leistungsbereitschaft, Teamfähigkeit, Umgangsformen sowie Zuverlässigkeit. Ausbildungsbetriebe legen gerade bei schulisch gering qualifizierten Jugendlichen einen besonderen Wert auf derartige Kompetenzen und Eigenschaften. Insbesondere das Arbeits- und Sozialverhalten spielt bei der Vergabe von Ausbildungsplätzen eine zentrale Rolle (vgl. Kohlrausch/Solga 2012). Dementsprechend zielen auch berufsvorbereitende Maßnahmen auf die Förderung derartiger Tugenden, die nicht losgelöst von den Geschlechterkonstruktionen der Jugendlichen betrachtet werden können. Um Letzteren ein möglichst realitätsnahes Lernen zu ermöglichen, arbeiten berufsvorbereitende Bildungsgänge eng mit Betrieben zusammen und sehen mehr oder weniger umfangreiche Praktikumsphasen vor.

\section{Männlichkeit im institutionellen Kontext von Schule und Berufsvorbereitung}

Im vorliegenden Beitrag wird die interaktive Herstellung von Männlichkeit im Zuge der Förderung von Ausbildungsreife fokussiert und davon ausgegangen, dass Männlichkeit ,erst in seinem Vollzug in Körperhaltungen, dem Umgang mit kulturellen Objekten, in Ritualen, Alltagstheorien und Institutionen hervorgebracht und identifizierbar [wird]" (Tervooren 2007: 88). In pädagogischen Institutionen lässt sich binnenmännliche Über- und Unterordnung, wie Connell (1999) sie beschreibt, beobachten, z.B. in Männlichkeitsinszenierungen von Schülern (vgl. Budde 2005). Körperbetonte Praktiken wie Kleidung und Haarstyling organisieren soziale Ein- und Ausschlüsse unter Jungen (vgl. Faulstich-Wieland/Weber/Willems 2004: 111ff.). Das ,doing masculinity، von sozial randständigen jungen Männern, wie sie an der Berufsvorbereitung teilnehmen, erscheint tendenziell negativ: So ist z.B. vom ,double looser ' die Rede, dessen Hauptproblem nicht biographische Unsicherheit sei, sondern „die Diskrepanz aus überzogenen Zukunftsvorstellungen und einer anders erlebten eigenen Realität" (Budde 2008: 34). Lehrkräfte in Berufsvorbereitungsklassen nehmen männliche Schüler angesichts von Verhaltens- und Disziplinproblemen als eine besondere Belastung wahr (vgl. Thielen 2013: 50).

Die Förderung von Ausbildungsreife erweist sich demzufolge bei männlichen Schülern als eine besondere Herausforderung. Die berufsbildende Schule nimmt unweigerlich Einfluss auf die Männlichkeitsinszenierungen der Jugendlichen. Dies geschieht allerdings nicht in Form gezielt geschlechtsbezogener didaktischer Interventionen - etwa im Sinne pädagogischer Jungen- 
arbeit -, sondern in nicht bewusst intendierten und damit unspezifischen Wirkungen der Institution im Sinne eines, heimlichen Lehrplans“ (vgl. Merkens 2006: 37). In diesem Zusammenhang ist von Bedeutung, dass sich die Akteure in der Bildungsinstitution als Rollenträger begegnen - also als Lehrer und Schüler - und dass die institutionell definierte Rolle deren Handlungsmöglichkeiten reglementiert (vgl. ebd.: 31f.). Zugleich sind jedoch die Beteiligten auch in ihren Geschlechterrollen präsent, wobei sich die neuere Männlichkeitsforschung meist am auf Bourdieu zurückgehenden Konzept des Geschlechterhabitus orientiert. Diesem zufolge wird Männlichkeit über alltägliche routinierte Handlungen inkorporiert und prägt damit den Habitus einer Person, also deren Denk-, Fühl-, Wahrnehmungs- und Handlungsschemata (vgl. Meuser 1998).

Das Handeln des einzelnen Akteurs in der Berufsvorbereitung ist somit zugleich durch die institutionelle Rolle (als Lehrer bzw. Schüler) und den vergeschlechtlichen Habitus (als Mann bzw. männlicher Jugendlicher) konstituiert. Allerdings manifestiert sich der männliche Habitus der Beteiligten in sehr unterschiedlichen Ausprägungen, die u.a. vom sozialen Milieu, der Generationenzugehörigkeit, der familiären Sozialisation sowie von lebensweltlichen Erfahrungshintergründen abhängen (vgl. ebd.: 115). Ein in der Regel bürgerlich konnotierter Habitus der Lehrkräfte prägt deren Sicht auf das sozialrandständige ,doing masculinity ${ }^{`}$ der Schüler. Die divergierenden Habitusausprägungen treffen im schulischen Alltag aufeinander und ziehen Konflikte nach sich. Dabei ist entscheidend, dass im institutionellen Kontext die normativen Männlichkeitserwartungen der Lehrkräfte hegemonial sind ${ }^{1}$ : Sie bestimmen über die Legitimität von Männlichkeitsinszenierungen und sanktionieren in ihrer mit Macht ausgestatteten Rolle solche Praktiken, die sich aus institutioneller Sicht als Störungen erweisen - in der binnenmännlichen Jugendkultur der Klasse jedoch möglicherweise Anerkennung und Zugehörigkeit sichern.

Angesichts umfangreicher Betriebspraktika und enger Kooperationen zwischen berufsbildender Schule und Betrieben verkompliziert sich das binnenmännliche Beziehungsgeflecht der Berufsvorbereitung: Zwar ist auch ein Praktikumsbetrieb eine pädagogische Institution, jedoch verfolgt diese, anders als die Schule, noch weitere nicht pädagogische Ziele - insbesondere ökonomische Interessen, mit Merkens (2006: 26) lässt sich von einer institutionellen Doppelfunktion sprechen. In den Firmen können demzufolge andere Männlichkeitserwartungen hegemonial sein als in der Schule. So sind in der Berufswelt je nach Beruf sehr spezielle und meist implizite Körperkonstruktionen notwendig (vgl. Villa 2007: 21). Da die Berufsvorbereitung die Schüler über das Betriebspraktikum in Ausbildung vermitteln möchte, spielen

1 In einer Gesellschaft existieren grundsätzlich verschiedene Versionen hegemonialer Männlichkeit, die jedoch jeweils nur in bestimmten sozialen Kontexten hegemonial sind (Scholz 2004: 47). 
derartige betriebliche Erwartungen auch im institutionellen Alltag der berufsbildenden Schule eine entscheidende Rolle und prägen die pädagogischen Praktiken zur Förderung von Ausbildungsreife. Letztere beziehen sich nicht zuletzt auch auf die Geschlechterkonstruktionen der Schüler.

\section{Ethnographie pädagogischer Praktiken zur Förderung von Ausbildungsreife in der Berufsvorbereitung}

Die im Anschluss skizzierten pädagogischen Bearbeitungsweisen von Männlichkeit wurden in einer ethnographischen Untersuchung zur Berufsvorbereitung als Teil pädagogischer Praktiken sichtbar, mittels derer die Institution auf die pädagogische Förderung von Ausbildungsreife ihrer Schülerschaft zielt. Während ethnographische Forschungsstrategien in der Schulpädagogik weitverbreitet sind (vgl. Heinzel 2010), finden sie in der Berufspädagogik bislang nur wenig Anwendung, mit dem Effekt, dass man meist nur vermittelt über Interviews mit den Akteuren Zugang zum Alltagsgeschehen in Institutionen der Berufsvorbereitung erhält (vgl. Giese 2011). Die Fokussierung auf narrative Verfahren erweist sich jedoch als problematisch, da ein großer Teil der alltäglichen Praktiken den Akteuren selbst gar nicht bewusst ist und demzufolge auch nicht ohne weiteres verbalisiert werden kann. Vor dem Hintergrund, dass Praktiken als typisierte, routinisierte und sozial ,verstehbare' Bündel von Aktivitäten verstanden werden, konzentriert sich praxeologische Forschung auf die Materialität des Sozialen bzw. des Kulturellen. Zwei Dimensionen sind dabei im Aufmerksamkeitsfokus: Menschliche Körper und Artefakte bzw. deren sinnhafter Gebrauch(vgl. Reckwitz 2003: 289ff.).

Um den pädagogischen Praktiken zur Förderung von Ausbildungsreife auf die Spur zu kommen führte ich qualitative Experteninterviews mit Lehrkräften und Gruppendiskussionen mit Schülern in Ergänzung zu teilnehmender Beobachtung am Unterricht sowie zu Tonmitschnitten einzelner Unterrichtsstunden. ${ }^{2}$ Hierzu habe ich die Berufsvorbereitungsklasse an einer beruflichen Schule in einer westdeutschen Großstadt zu unterschiedlichen, über das gesamte Schuljahr verteilten Zeiten ethnographisch begleitet. ${ }^{3}$ Die unter-

2 Bei der Datenauswertung orientiere ich mich an der Grounded Theorie nach Glaser und Strauss sowie der Dokumentarischen Methode nach Bohnsack.

3 Um sicherzustellen, dass es sich bei den beobachteten Phänomenen um typische Ereignisse handelt, habe ich in den letzten Wochen des vorangegangenen Schuljahres sowie in den ersten Wochen im nachfolgenden Schuljahr den Unterricht von zwei weiteren Klassen beobachtet. 
suchte Lagerlogistikklasse setzte sich ausnahmslos aus männlichen Schülern zusammen, die zwischen 16 und 19 Jahren alt waren. Über das gesamte Schuljahr waren zwanzig Jugendliche in der Klasse, von denen dreizehn einen Migrationshintergrund hatten ${ }^{4}$. Die Familien zeichnen sich durch einen niedrigen Sozialstatus aus: Nur die Hälfte der Eltern verfügt über eine abgeschlossene Berufsausbildung. Die Väter gehen Bauberufen nach, arbeiten im Transport, der Lagerlogistik und der Gebäudetechnik oder sind in der Reinigung und Abfallwirtschaft tätig. Die Mütter sind in der Reinigung, der Gastronomie und im Einzelhandel beschäftigt. Jeder fünfte Vater und jede vierte Mutter sind nicht erwerbstätig. Für die Klasse waren vier männliche Lehrkräfte sowie eine Sozialpädagogin und ein Sozialpädagoge - allesamt ohne Migrationshintergrund - zuständig. Im Feld wurde rasch offenkundig, dass eine Vielzahl der rekonstruierten pädagogischen Praktiken zur Förderung von Ausbildungsreife auf die Männlichkeit der Schüler rekurriert.

\section{Praktiken institutioneller Bearbeitung von Männlichkeit}

Die Thematisierung von Männlichkeit im Feld resultierte insbesondere aus der Beobachtung, dass die Vorbereitungsklassen an der untersuchten Berufsschule überwiegend bis ausschließlich von männlichen Schülern besucht werden. Die pädagogischen Akteure begründen dies domänenspezifisch mit dem Berufsfeld, so etwa ein interviewter Lehrer:

„Also erst mal bringt das so der fachliche Hintergrund nun mal mit sich, dass nun mal überwiegend Männer im Lager arbeiten. Wobei es gibt durchaus auch Fachlageristinnen. Lager sind ja teilweise heute hoch technisiert. Dann muss man teilweise ja gar nichts mehr schleppen. In einigen Lagern. Es gibt aber auch solche, die körperlich sehr anstrengend sind " (Lehrer).

Wenngleich sich die Lagerlogistik angesichts des technischen Fortschritts auch weiblichen Arbeitskräften geöffnet hat, markiert sie der Lehrer angesichts der auch gegenwärtig häufig noch zu verrichtenden körperlichen Tätigkeiten als eine männliche Domäne. Männlichkeit wird demnach über körperliche Stärke definiert. Viele der im institutionellen Alltag der Berufsvorbereitung sichtbar werdenden Praktiken zur Förderung von Ausbildungsreife beziehen sich auf den diskursiven Zusammenhang von Männlichkeit und

4 Bei den Herkunftsländern handelt es sich um Afghanistan, Ägypten, Aserbaidschan, Chile, Ecuador, Irak, Kosovo, Mazedonien, Serbien (zwei Schüler), Spanien, Türkei (zwei Schüler). Sechs Schüler verfügen über eigene Migrationserfahrung. 
körperlicher Arbeit. Berufsorientierung obliegt in dieser Perspektive die Hinführung zu manuellen Tätigkeiten.

\subsection{Orientierung auf manuelle Tätigkeiten}

Insbesondere zu Schuljahresbeginn artikulieren die Schüler Unsicherheiten im Hinblick auf ihre Berufswahl und verweisen dabei auch auf den ihrer Einschätzung nach geringen gesellschaftlichen Status von Lagerarbeitern. Hierzu exemplarisch eine Sequenz aus einem Unterrichtsgespräch, in dem die Berufswünsche der Schüler abgefragt werden:

„L: Okay, Serdar ${ }^{5}$, was für nen Berufswunsch hast du?

S: Ich weiß nicht. Lager? Ich weiß es echt nicht. Ich muss mir erst mal alles angucken. Ich bin mir nicht sicher, für was für einen Berufich mich entscheide.

L: Noch nicht sicher beziehungsweise du hast noch keine Orientierung im Augenblick, oder?

S: Was mir Spaß macht halt!

L: Das weißt du aber noch nicht, was dir Spaß machen würde?

S: Ja. Meistens ist ja echt sowieso der Job, der dir Spaß macht, am besten!

L: Genau! Deswegen jetzt die Frage.

S: Ich weiß ja nicht, wie das im Lager ist. Vielleicht muss man ja alles tragen wie ein Packesel oder so was. Das würde mir nicht Spaß machen. “"

Serdar begründet seine offenkundig noch nicht geklärte Berufswahl explizit mit der körperlichen Belastung (,alles tragen“), die seiner Einschätzung nach mit der Tätigkeit im Lager verbunden sein könnte. Im Bild des „Packesels“ manifestiert sich die Geringschätzung körperlicher Arbeit, repräsentiert doch der Esel in Fabelgeschichten zumeist negativ konnotierte Persönlichkeitseigenschaften wie Dummheit oder Sturheit. Als Lasttier gilt er jedoch angesichts seiner Hartnäckigkeit als nützlich, womit auf die Funktion des männlichen Körpers als zu disziplinierendes Instrument harter körperlicher Arbeit verwiesen ist (vgl. Meuser 2007: 153). In Serdars Deutung offenbart sich die kulturell verankerte Dualität von körperlicher und geistiger Arbeit - letztere wird jemandem, der als kognitiv minderbegabt gilt, nicht zugetraut. Die unterschiedlichen Arbeitsformen stehen in einem hierarchischen Verhältnis zueinander und organisieren binnenmännliche Ungleichheiten: Gebildete Männer im bürgerlichen Milieu sind in ihrer beruflichen Leistung nicht auf die Bereitstellung ihrer Körperkraft angewiesen und grenzen sich über jenes Privileg von Männern aus unteren Sozialmilieus ab (vgl. Meuser 2007: 156). Serdar indes lehnt eine Tätigkeit, die sich auf die stupide Verrichtung manueller Tätigkeit beschränkt, offenkundig ab. Der Lehrer echauffiert sich kurze

$5 \quad$ Alle im Beitrag zitierten Namen sind geändert. 
Zeit später über die seiner Ansicht nach unrealistischen Berufsvorstellungen der Schüler (genannt wurden Kfz-Mechatroniker, Einzelhandelskaufmann, Fachinformatiker, Bürokaufmann, Tischler, Manager bei Mercedes und Popstar):

„Ihr sollt einen Ausbildungsplatz finden! Und einen Ausbildungsplatz, der zu euch passt! Ein Praktikum machen, was zu euch passt! Jemand, der ein paar Jahre nicht in der Schule war und dann Bürokaufmann werden will, was vom Kopf jetzt ziemlich anspruchsvoll ist, macht keinen Sinn! So ein Quatsch" (Lehrer).

Die Ansprache des Lehrers lässt sich als Appell zu einer von den Jugendlichen zu erbringenden Aspirationsabkühlung interpretieren. Der Abgleich ihrer individuellen Voraussetzungen mit beruflichen Anforderungen verlangt angesichts des mangelnden Bildungserfolgs eine Korrektur der beruflichen Ziele nach ,unten': Allenfalls niedrige berufliche Positionen scheinen erreichbar. Diesbezüglich rekurriert auch der Lehrer auf die Differenz von körperlicher und geistiger Arbeit (,vom Kopf her") und schließt zumindest anspruchsvolle kognitive Tätigkeiten aus. Zugleich werden die Schüler in ihren zuvor genannten beruflichen Wünschen, Träumen und Zukunftsvorstellungen entwertet und beschämt (,So ein Quatsch!“).

\subsection{Herausforderung männlicher Härte}

Die Vorbereitung auf manuelle Tätigkeiten geht mit pädagogischen Praktiken zur Herausforderung männlicher Härte und Maskulinität einher. So begründet ein Lehrer die Tatsache, dass etliche Wochen nach Schuljahresbeginn nur wenige Jugendliche im Praktikum sind, damit, dass Betriebe ,robuste Praktikanten“ bevorzugten, viele der Schüler aber noch zu „flauschig“ seien. Die mangelnde Betriebsreife der Jugendlichen wird somit geschlechtsspezifisch interpretiert und mit fehlender männlicher Härte konnotiert. Auf Nachfrage bezieht der Lehrer die nötige „Robustheit“ auf das „raue Arbeitsklima“ in Betrieben. Fehlgeschlagene Vermittlungsversuche in ein Praktikum und Praktikumsabbrüche werden in Interviews häufig mit unzureichender körperlicher Belastung begründet. Entsprechend werden die Schüler in unterrichtlichen Interaktionen zu männlicher Härte angehalten. Besonders deutlich zeigt sich dies am einmal wöchentlich stattfindenden Werkstatttag, an dem die Schüler angeleitet von zwei Lehrern in kleinen Teams in der Lagerlogistik eingesetzte Europoolpaletten und Transportkisten aus Holzbrettern herstellen. Hierbei wurde folgende Szene beobachtet:

„Herr Peters fordert den gerade erst zum Unterricht erschienenen Brandon auf, zunächst mit ihm in den Keller zu gehen, um von dort aus eine schon fertige Europalette zu holen, die dem Schüler als Modell dienen soll. Brandon ist heute zum ersten 
Mal in der Werkstatt. Schüler und Lehrer verlassen daraufhin gemeinsam die Werkstatt und kommen nach einer kurzen Zeit mit einer Europalette in den Händen zurück. Nachdem sie diese in den Raum gebracht und auf dem Boden abgelegt haben, gibt Brandon schnaufend und schwer atmend zu verstehen, dass die Palette, ganz schön schwer' sei, woraufhin ihm Herr Peters entgegnet: , Genau Brandon, das macht Muskeln! " “

Der in der Werkstatt noch unerfahrene Brandon zeigt sich über das Gewicht der Palette (etwa zwanzig Kilo) erstaunt und bringt auch nonverbal zum Ausdruck, dass er das Tragen der selbigen als körperlich anstrengend empfindet. Mit der pädagogischen Intervention wird dem Schüler also nicht nur der Aufbau einer Europalette plastisch verdeutlicht, sondern zugleich auch ein leibhaftiger Einblick in das Tätigkeitsprofil eines Lageristen eröffnet, zu dem auch das Bewegen von schweren Gegenständen durch den Einsatz körperlicher Kraft gehört. In dem Versuch des Lehrers, dem Schüler die körperliche Anstrengung durch das prophezeite Muskelwachstum schmackhaft zu machen, wird abermals auf Männlichkeit Bezug genommen - ein muskulöser Körper repräsentiert Virilität - und dabei zugleich Anschluss an die männlichen Körperpraktiken der Schüler gesucht. Einige von diesen besuchen in ihrer Freizeit ein Fitnessstudio. Allerdings dient das dort betriebene Krafttraining nicht der Instrumentalisierung des männlichen Körpers, sondern dessen ästhetischer Gestaltung. Dieser Unterschied ist den Jugendlichen durchaus bewusst. Auch in weiteren Szenen werden Schüler durch den Verweis auf ihren männlichen Status und die daraus resultierende Verpflichtung zu männlicher Härte motiviert. So meint ein Lehrer zu einem die anstrengende Arbeit beklagenden Schüler: „Ach komm, was uns nicht umbringt, macht uns noch härter!" Der Pädagoge verweist auf die gemeinsame Zugehörigkeit zum männlichen Geschlecht (,uns") und damit auf die Bedeutung von hegemonialen Männlichkeitserwartungen, die Schüler und Lehrer gleichermaßen zu erfüllen haben.

\subsection{Sanktionierung unmännlicher Schwäche}

Die Förderung männlicher Härte geht mit pädagogischen Praktiken einher, die unerwünschte Verhaltensweisen von Schülern als ,unmännlich“ und ,schwach' konnotieren. Exemplarisch hierzu eine weitere Szene aus der Werkstatt, in der ein Schüler gemeinsam mit einem Lehrer an einem Holzverschlag arbeitet, der dem Transport eines Kompressors dienen soll. Der Schüler unterbricht die Arbeit nun schon zum wiederholten Male, obwohl gerade erst große Pause war:

„Cengiz beklagt, dass er am Vorabend im Fitnessstudio war und nun Muskelkater habe. Die Arme täten ihm weh. Herr Peters ermuntert den Schüler dennoch weiter mit 
dem Kollegen Schuster am Holzverschlag zu arbeiten. Kurz darauf geht Cengiz jedoch zum Eingangsbereich, während Herr Schuster alleine weiterarbeitet. Der Schüler packt gemächlich eine große Flasche Mezzo Mix aus seinem Rucksack. Er nimmt die Flasche, schraubt den Verschluss auf und setzt gerade zum Trinken an, als Herr Schuster, der den Schüler offenbar beobachtet, laut und in ironischem Ton durch die Werkstatt ruft: ,Vorsicht, nicht die Flasche zu doll anheben, du hast ja Muskelkater! Nachher tut es noch weh! 'Einige Schüler lachen laut."

Die pädagogische Intervention lässt sich als eine erzieherische Praktik verstehen, mittels derer mangelndes Durchhaltevermögen - eine für Betriebe wesentliche Dimension von Ausbildungsreife - sanktioniert wird. Cengiz, der die Arbeit am Holzverschlag offenkundig als zu anstrengend empfindet, ist bemüht, trotz seiner vielen Pausen seinen männlichen Status zu sichern. So verweist er mit der expliziten Erwähnung seines Muskelkaters auf ein vermeintlich besonders hartes Krafttraining am Vortag. Die Strategie des Schülers scheitert jedoch, da sich Herr Schuster mit seiner lautstarken und ironischen Kommentierung publikumswirksam über Wehleidigkeit und damit fehlende Härte von Cengiz mokiert und hierfür ein bestätigendes, den Jugendlichen in seiner Männlichkeit zugleich beschämendes kollektives Lachen von Mitschülern erntet. Diese pädagogische Praktik ist gerade deshalb wirkungsvoll, da sie Effekte in der binnenmännlichen Gruppendynamik der Schüler nach sich zieht. Cengiz' Status in der Peergroup steht zur Disposition. Dies zeigt sich wenige Minuten später, als Mitschüler beginnen, Cengiz angesichts seiner offenkundig gewordenen Schwäche zu provozieren. So unterstreicht auch Mohammed die unzureichende Arbeitsleistung von Cengiz, indem er sich demonstrativ neben ihn stellt und ihn antreibt: „Na los Cengiz! Heb mal den Kompressor an!“ Auch Sven stimmt ein: „Na los Cengiz!“ Die Mitschüler beteiligen sich demzufolge an der Überwachung der in der Institution hegemonialen Männlichkeitswartungen und erinnern Cengiz an deren Gültigkeit. Cengiz indes reagiert sichtlich gereizt und kontert, indem er die Körperkraft von Sven infrage stellt: „Ich will mal sehen, ob du ihn [den Kompressor] packst!“ Die pädagogische Praktik aktiviert demnach binnenmännliche Konkurrenz in Form eines Wettstreits um körperliche Stärke, ein Männlichkeitsideal, das auch in den jugendkulturellen Praktiken der Schüler hegemonial ist. In Bezug auf die ökonomisch intendierte Instrumentalisierung des männlichen Körpers zur Verrichtung körperlicher Arbeit kann sich ein solcher Wettbewerb durchaus als funktional erweisen, etwa wenn sich Mitarbeiter in einem Betrieb über den konkurrierenden Vergleich ihrer Arbeitsleistung gegenseitig anspornen und damit die Produktivität steigern. 


\section{Fazit}

Der exemplarische Ausschnitt aus dem unterrichtlichen Alltag der Berufsvorbereitung gibt einen Einblick, wie sich die institutionellen Praktiken zur Förderung von Ausbildungsreife im Berufsfeld Lagerlogistik in besonderer Weise auf die Männlichkeitskonstruktionen der Schüler beziehen. Infolge ihres mangelnden schulischen Bildungserfolgs sehen sich die Heranwachsenden mit dem Eintritt in die Berufsbildung am unteren Ende der Berufshierarchie. Ihre Berufswahlentscheidung geht angesichts der begrenzten Optionen mit negativen Auswirkungen auf ihr Selbstwertgefühl und ihren Zukunftsoptimismus einher (vgl. Scherr 2012). Die im binnenmännlichen Geschlechterverhältnis untergeordnete Position manifestiert sich in der Zuweisung in ein Berufsfeld, das an die Instrumentalisierung des jugendlichen Körpers für die Verrichtung manueller Tätigkeiten geknüpft ist. Die institutionelle Vorbereitung auf eine Berufsausbildung in der Lagerlogistik (Fachlagerist oder Fachkraft für Lagerlogistik) vollzieht sich demzufolge über Praktiken zur Förderung männlicher Härte in Gestalt von körperlicher Stärke, Ausdauer und Disziplin. Hiervon abweichende Verhaltensweisen werden institutionell als unmännlich markiert und damit geschlechtsbezogen sanktioniert. In den rekonstruierten Praktiken zur Förderung von Ausbildungsreife spiegelt sich demzufolge eine Normalisierungsmacht wider, die mit der Anpassung an traditionelle Männlichkeitserwartungen einhergeht, die auch in männlichen Jugendkulturen wirksam sind. Vor diesem Hintergrund vollzieht sich die Durchsetzung der hegemonialen Männlichkeitserwartungen in der Institution der berufsbildenden Schule gerade nicht entlang einer klar zu ziehenden ,Frontlinie' zwischen Lehrkräften und Schülern. Vielmehr setzen einige der rekonstruierten Praktiken zur Förderung von Ausbildungsreife explizit die Mitwirkung der männlichen Peergroup voraus, die von den Lehrkräften mit der Überwachung der Einhaltung hegemonialer Männlichkeitsideale ,beauftragt $^{`}$ wird. Dabei werden die Schüler gezielt in ihrer Geschlechtlichkeit adressiert und an die daraus resultierende kollektive Verantwortung für männliche Arbeitstugenden erinnert. Da körperliche Stärke auch in den Peerkontexten der Jugendlichen eine entscheidende Rolle spielt und dort überund untergeordnete Positionen organisiert, wird verstehbar, weshalb sich Interaktionen beobachten lassen, in denen Schüler die Schule bei der Umsetzung pädagogischer Praktiken zur Förderung der Ausbildungsreife unterstützen. Im Verhältnis zu den Lehrkräften erscheinen die Jugendlichen dabei in der Rolle einer komplizenhaften Männlichkeit, die sich im Zuge der Abwertung des ,unmännlichen' Verhaltens von Mitschülern ihrer eigenen Männlichkeit rückversichern kann. Zugleich wird über Prozesse der Beschämung soziale Exklusion im binnenmännlicher Geschlechterverhältnis der Klasse organisiert und die Stabilisierung hegemonialer Männlichkeitserwartungen 
gesichert. Im Hinblick auf die Berufswahlentscheidung von Jugendlichen in berufsvorbereitenden Bildungsgängen und die dort bereits einsetzende berufliche Sozialisation erscheinen die Befunde bemerkenswert. Während sich junge Männer aus der Arbeiterklasse in der klassischen ethnographischen Studie von Paul Willis (1979) den männlichen Habitus des Industriearbeitermilieus in Opposition gegen die Institution Schule durch die Etablierung einer adoleszenten Gegenkultur ,selbst' aneignen, verbünden sich im ethnographierten berufsvorbereitenden Bildungsgang pädagogische Praktiken zur Förderung von Ausbildungsreife mit jugendkulturellen Praktiken zur Herstellung von Männlichkeit, um das Ziel der Aneignung eines für die manuellen Tätigkeiten im Berufsfeld der Lagerlogistik funktionalen Geschlechtshabitus zu erreichen. Das pädagogische Arrangement der Berufsvorbereitung, das auf die Förderung von Ausbildungsreife zielt, lässt sich in männlich dominierten Berufen demnach auch als eine Schule von Männlichkeit verstehen. In einer segmentierten Berufswelt, in der schulisch gering qualifizierten Jugendlichen nur eingeschränkte berufliche Optionen offenstehen, erweist sich dies durchaus als funktional: Wenngleich die erreichbaren Berufsfelder nur über ein bedingtes soziales Prestige verfügen, sichern sie den Jugendlichen zumindest die Zugehörigkeit zu in unteren sozialen Milieus anerkannter Männlichkeit, die sich insbesondere auch über körperliche Stärke definiert. Die institutionellen Praktiken zur Förderung der Ausbildungsreife zeichnen sich folglich durch eine grundsätzliche Ambivalenz aus: Einerseits zielen sie auf eine Erweiterung der Handlungsfähigkeit der Schüler im Hinblick auf die Bewältigung der realen und zweifelsohne harten Arbeitsbedingungen im männlich dominierten Berufsfeld der Lagerlogistik. Andererseits begünstigen sie damit zugleich eine Begrenzung der prinzipiellen Vielfalt an männlichen Verwirklichungs- und Ausdrucksformen und unterstützen die Reproduktion traditioneller Männlichkeitsnormen.

\section{Literatur}

Baethge, M. (2010): Neue soziale Segmentationsmuster in der beruflichen Bildung. In: Krüger, H./Rabe-Kleberg, U./Kramer, R./Budde, J.(Hrsg.): Bildungsungleichheit revisited. Bildung und soziale Ungleichheit vom Kindergarten bis zur Hochschule. Wiesbaden: VS-Verlag für Sozialwissenschaften, S. 275-298.

Beicht, U./Eberhard, V. (2013): Ergebnisse einer empirischen Analyse zum Übergangssystem auf der Basis der BIBB-Übergangsstudie 2011. In: Die Deutsche Schule 105, 1, S. 10-27.

Beicht, U./Walden, G. (2012): Berufswahl und geschlechtsspezifische Unterschiede beim Zugang zu betrieblicher Ausbildung. In: Zeitschrift für Berufs- und Wirtschaftspädagogik 108, 4, S. 491-510. 
Budde, J. (2005): Männlichkeit und gymnasialer Alltag. Doing gender in heutigen Bildungsinstitutionen. Bielefeld: transcript.

Budde, J. (2008): Bildungs(miss)erfolge von Jungen und Berufswahlverhalten bei Jungen/männlichen Jugendlichen. Bonn und Berlin: Bundesministerium für Bildung und Forschung.

Bundesagentur für Arbeit (Hrsg.) (2009): Nationaler Pakt für Ausbildung und Fachkräftenachwuchs in Deutschland. Kriterienkatalog zur Ausbildungsreife. Nachdruck 2009. Online: http://www.arbeitsagentur.de/zentraler-Content/ Veroeffentlichungen/Ausbildung/Kriterienkatalog-zur-Ausbildungsreife.pdf

Connell, R. (1999): Der gemachte Mann. Konstruktion und Krise von Männlichkeiten. Opladen: Leske + Budrich.

DHIK - Deutscher Industrie- und Handelskammertag (Hrsg.) (2012): Ausbildung 2012. Ergebnisse einer DIHK-Online-Unternehmensbefragung. Berlin.

Faulstich-Wieland, H./Weber, M./Willems K. (2004): Doing Gender im heutigen Schulalltag. Empirische Studien zur sozialen Konstruktion von Geschlecht in schulischen Interaktionen. Weinheim u. München: Juventa.

Giese, J. (2011): „Besser als zu Hause rumsitzen“. Zur Wahrnehmung und Bewältigung interner Ausgrenzung im Übergangssystem zwischen Schule und Beruf. Bad Heilbrunn: Klinkhardt.

Heinzel, F. (2010): Ethnographische Untersuchung von Mikroprozessen in der Schule. In: Heinzel, F./Thole, W./Cloos, P./Köngeter, S. (Hrsg.): „Auf unsicherem Terrain“. Ethnographische Forschung im Kontext des Bildungs- und Sozialwesens. Wiesbaden: VS-Verlag für Sozialwissenschaften, S. 39-47.

Merkens, H. (2006): Pädagogische Institutionen. Pädagogisches Handeln im Spannungsfeld von Individualisierung und Organisation. Wiesbaden: VS Verlag für Sozialwissenschaften.

Meuser, M. (1998): Geschlecht und Männlichkeit. Soziologische Theorie und kulturelle Deutungsmuster. Opladen: Leske + Budrich.

Meuser, M. (2007): Männerkörper. Diskursive Anregungen und habitualisierte Praxis. In: Bereswill, M./Meuser, M./Scholz, S. (Hrsg.): Dimensionen der Kategorie Geschlecht: Der Fall Männlichkeit. Münster: Westfälisches Dampfboot, S. 152-168.

Müller, S./Rebmann, K. (2008): Ausbildungsreife von Jugendlichen im Urteil von Lehrkräften. In: Zeitschrift für Berufs- und Wirtschaftspädagogik. 104. Bd., 4, S. 573-589.

Reckwitz, A. (2003): Grundelemente einer Theorie sozialer Praktiken. Eine sozialtheoretische Perspektive. In: Zeitschrift für Soziologie32, S. 282-301.

Scherr, A. (2012): Hauptsache irgendeine Arbeit? Die Bedeutung von Ausbildung und Erwerbsarbeit für benachteiligte Jugendliche. In: Mansel, J./Speck, K. (Hrsg.): Jugend und Arbeit. Empirische Bestandsaufnahme und Analysen. Weinheim u. München: Beltz Juventa, S. 63-76.

Scholz, S. (2004): Männlichkeit erzählen. Lebensgeschichtliche Identitätskonstruktionen ostdeutscher Männer. Münster: Westfälisches Dampfboot.

Schroeder, J./Thielen, M. (2009): Das Berufsvorbereitungsjahr. Eine Einführung. Stuttgart: Kohlhammer.

Tervooren, A.(2007): Männlichkeiten und Sozialisation. Die allmähliche Verfestigung der Körper. In: Bereswill, M./Meuser, M./Scholz, S. (Hrsg.): Dimensionen der Kategorie Geschlecht: Der Fall Männlichkeit. Münster: Westfälisches Dampfboot, S. 84-100. 
Thielen, M. (2013): Zweijährige Berufsvorbereitung. Eine Verbleibstudie zum Schulversuch "Gestrecktes Berufsvorbereitungsjahr" in Sachen. Bad Heilbrunn: Klinkhardt.

Villa, P.(2007): Der Körper als kulturelle Inszenierung und als Statussymbol. In: Politik und Zeitgeschichte 18/2007, S. 18-26.

Willis, P. (1979): Spaß am Widerstand. Gegenkultur in der Arbeiterschule. Frankfurt am Main: Syndikat 